\title{
Vacuum Energy as Spectral Geometry ${ }^{\star}$
}

Stephen A. FULLING

Department of Mathematics, Texas A\&M University, College Station, Texas, 77843-3368, USA

E-mail: fulling@math.tamu.edu

URL: http://www. math.tamu.edu/ fulling/

Received June 21, 2007, in final form September 14, 2007; Published online September 26, 2007

Original article is available at http://www.emis.de/journals/SIGMA/2007/094/

\begin{abstract}
Quantum vacuum energy (Casimir energy) is reviewed for a mathematical audience as a topic in spectral theory. Then some one-dimensional systems are solved exactly, in terms of closed classical paths and periodic orbits. The relations among local spectral densities, energy densities, global eigenvalue densities, and total energies are demonstrated. This material provides background and motivation for the treatment of higher-dimensional systems (self-adjoint second-order partial differential operators) by semiclassical approximation and other methods.
\end{abstract}

Key words: Casimir; periodic orbit; energy density; cylinder kernel

2000 Mathematics Subject Classification: 34B27; 81Q10; 58J50

\section{Introduction}

Vacuum energy is a concept arising in quantum field theory, with observable consequences of considerable current interest in physics $[13,52,50,7,41]$. Here, however, I treat it as a purely mathematical problem, an underdeveloped aspect of the spectral theory of self-adjoint secondorder differential operators.

Vacuum energy is related to the oscillatory terms in the spectral density, which are associated with the periodic orbits in the classical mechanics determined by the operator as Hamiltonian $[1,2,15,36,19,16,9]$. The information concerning these oscillations is lost from the muchstudied short-time expansion of the heat kernel [51, 44, 35, 32, 9, 45], but some of it remains in the asymptotics of another integral kernel, the cylinder kernel, which includes the vacuum energy directly $[48,5,37,29,26,27]$. The local density of vacuum energy is related analogously to a local spectral density, associated with classical paths that are closed but not necessarily periodic. Thus the vacuum energy and its density are probes of the detailed geometry associated with the operator.

The connection between closed classical paths and Casimir energy is implicit in the two papers of Balian and Duplantier [3], which treat those two aspects of the electromagnetic field separately. In recent years it has become a serious tool for the calculation of vacuum energies $[43,57,49,27,54,55,56,39]$. In one spatial dimension it reduces to the classical method of images, which has been applied to Casimir calculations since at least 1969 [11].

The main purpose of this article is to demonstrate in detail, for the simplest one-dimensional models, the connections among closed orbits, spectral densities, and vacuum energy densities, as well as the corresponding global quantities. Sections 3 and 4 are based on research notes that I have used for some years with my students and collaborators; the fact that they have not been published and available for citation has become increasingly inconvenient. First, however, in Section 2 I present the basics of vacuum energy in a more general context to a mathematical

${ }^{\star}$ This paper is a contribution to the Proceedings of the 2007 Midwest Geometry Conference in honor of Thomas P. Branson. The full collection is available at http://www.emis.de/journals/SIGMA/MGC2007.html 
audience. This material is based on talks given at the Workshop on Semiclassical Approximation and Vacuum Energy at Texas A\&M University in January, 2005, and the Workshop on Spectral Theory and Its Applications at the Isaac Newton Institute, University of Cambridge, in July, 2006. I expect to publish elsewhere [30] a more complete discussion of the physics of the subject, still for a mathematical audience, along with a review of the recent work on vacuum energy in quantum graphs [59].

\section{Vacuum energy (and energy density) in general}

\subsection{Spectral theory}

Let $H$ be a second-order, elliptic, self-adjoint partial differential operator, on scalar functions, in a $d$-dimensional region $\Omega$. The prototype situation is a billiard:

$$
H=-\nabla^{2}, \quad \Omega \subset \mathbf{R}^{d}
$$

with boundary conditions that make the operator self-adjoint (such as the Dirichlet condition, $u=0$ on $\partial \Omega$ ). The treatment can be generalized to the electromagnetic field (vector functions) which is the case of greatest physical interest - and to other boundary conditions, to Riemannian manifolds (Laplace-Beltrami operators), potentials $\left(H=-\nabla^{2}+V(x)\right)$, applied magnetic fields (gauge-invariant Laplacians on vector bundles), etc. For simplicity, assume that the spectrum of $H$ is nonnegative and that if 0 is an eigenvalue, then the eigenfunction is constant. Of course, for precise theorems some smoothness hypotheses on the boundary, potential, etc. are needed.

For the moment let us assume that the spectrum is discrete, as will be so if $\Omega$ is a compact billiard. In this case a finite total vacuum energy is expected. We review the spectral decomposition and functional calculus. Let $\varphi_{n}$ be the normalized eigenfunctions:

$$
H \varphi_{n}=\lambda_{n} \varphi_{n}, \quad\left\|\varphi_{n}\right\|^{2}=\int_{\Omega}\left|\varphi_{n}(x)\right|^{2} d x=1
$$

Define $\omega_{n}=\sqrt{\lambda_{n}}$. Functions of the operator $H$ are defined by

$$
f(H) u \equiv \sum_{n=1}^{\infty} f\left(\lambda_{n}\right)\left\langle\varphi_{n}, u\right\rangle \varphi_{n}, \quad\left\langle\varphi_{n}, u\right\rangle \equiv \int_{\Omega} \overline{\varphi_{n}(x)} u(x) d x .
$$

At least formally, $f(H)$ is given by an integral kernel:

$$
f(H) u(x)=\int_{\Omega} G(x, y) u(y) d y, \quad G(x, y)=\sum_{n=1}^{\infty} f\left(\lambda_{n}\right) \varphi_{n}(x) \overline{\varphi_{n}(y)}
$$

In general $G$ is a distribution, but if $f$ is sufficiently rapidly decreasing, $G$ is a smooth function, and the trace is defined:

$$
\operatorname{Tr} G \equiv \int_{\Omega} G(x, x) d x=\sum_{n=1}^{\infty} f\left(\lambda_{n}\right) \equiv \operatorname{Tr} f(H)
$$

The prototype is the heat kernel, $G(x, y)=K(t, x, y)$, corresponding to the parametrized function $f_{t}(\lambda)=e^{-t \lambda}$. Then $u(t, x)=f_{t}(H) u_{0}$ solves

$$
\frac{\partial u}{\partial t}=-H u, \quad u(0, x)=u_{0}(x) .
$$


It is well known $[35,10,32,45]$ that $K$ for a billiard has the asymptotic expansion

$$
\operatorname{Tr} K=\sum_{n=1}^{\infty} e^{-t \lambda_{n}} \sim \sum_{s=0}^{\infty} b_{s} t^{(-d+s) / 2}
$$

where each term has a global geometrical significance - for instance, $b_{0}$ is proportional to the volume of $\Omega$. The inverse Laplace transform of the leading term in $\operatorname{Tr} K$ gives the leading behavior at large $\lambda$ of the density of eigenvalues, and the higher-order terms correspond similarly to lower-order corrections to the eigenvalue distribution, on the average [12, 40, 25]. (When $V(x)$ is a confining potential [36, 2, 9], $H=-\nabla^{2}+V$ may have discrete spectrum even though its spatial domain, $\Omega$, is not compact. In such a case the form of the asymptotic expansion of $\operatorname{Tr} K$, and the resulting asymptotic expansion of the eigenvalue distribution, may be rather different. For example, the eigenvalues of a one-dimensional harmonic oscillator are evenly spaced in $\lambda$, whereas those of a one-dimensional billiard are evenly spaced in $\omega$. .)

The cylinder kernel (also called Poisson kernel), $T(t, x, y)$, corresponds to the parametrized function $f_{t}(\lambda)=e^{-t \sqrt{\lambda}}$. That is, $f_{t}(H) u_{0}$ is the solution of

$$
\frac{\partial^{2} u}{\partial t^{2}}=H u, \quad u(0, x)=u_{0}(x)
$$

that is well-behaved as $t \rightarrow+\infty$. ( $T$ is a boundary value of a derivative of the fundamental solution of the elliptic operator $H-\frac{\partial^{2}}{\partial t^{2}}$ in $\Omega \times \mathbf{R}$. It is the ("imaginary-time") analytic continuation of the time derivative of the Wightman function, a certain fundamental solution of the hyperbolic operator $H+\frac{\partial^{2}}{\partial t^{2}}$.) We have

$$
T(t, x, y)=\sum_{n=1}^{\infty} e^{-t \omega_{n}} \varphi_{n}(x) \overline{\varphi_{n}(y)}, \quad \operatorname{Tr} T=\int_{\Omega} T(t, x, x) d x=\sum_{n=1}^{\infty} e^{-t \omega_{n}} .
$$

As $t \downarrow 0$ one has the asymptotics [33, 29, 4]

$$
\operatorname{Tr} T \sim \sum_{s=0}^{\infty} e_{s} t^{-d+s}+\sum_{\substack{s=d+1 \\ s-d \text { odd }}}^{\infty} f_{s} t^{-d+s} \ln t
$$

as described in the following theorem. (For a more complete, but succinct, statement of the connection with Riesz means, see [27]. See also [14, 20, 21, 26].)

Theorem 1. The traces of the heat kernel and the cylinder kernel of a positive, self-adjoint, second-order linear differential operator in dimension $d$ have the asymptotic expansions (2.1) and (2.2), and precisely parallel expansions hold for the local (untraced) diagonal values of those kernels. The $b_{s}$ are proportional to coefficients in the high-frequency asymptotics of Riesz means of $N$ (or P) with respect to $\lambda$. The $e_{s}$ and $f_{s}$ are proportional to coefficients in the asymptotics of Riesz means with respect to $\omega$. If $d-s$ is even or positive,

$$
e_{s}=\pi^{-1 / 2} 2^{d-s} \Gamma((d-s+1) / 2) b_{s} .
$$

If $d-s$ is odd and negative,

$$
f_{s}=\frac{(-1)^{(s-d+1) / 2} 2^{d-s+1}}{\sqrt{\pi} \Gamma((s-d+1) / 2)} b_{s},
$$

but $e_{s}$ is undetermined by the $b_{r}$. 


\subsection{Vacuum energy}

We can now define the vacuum energy as the coefficient with $d-s=-1$,

$$
E \equiv-\frac{1}{2} e_{1+d}
$$

Formally, $E$ is the "finite part" of

$$
\frac{1}{2} \sum_{n=1}^{\infty} \omega_{n}=-\left.\frac{1}{2} \frac{d}{d t} \sum_{n} e^{-\omega_{n} t}\right|_{t=0}
$$

(When $f_{1+d} \neq 0, E$ is actually defined only modulo a multiple of $f_{1+d}$, because of the scale ambiguity in the argument of the logarithm. That complication will not arise in the problems studied in this paper.)

The prototype example is

$$
\Omega=(0, L), \quad H=-\frac{d^{2}}{d x^{2}} ; \quad \omega_{n}=\frac{n \pi}{L}, \quad \varphi_{n}(x)=\sin \left(\frac{n \pi x}{L}\right) .
$$

One can evaluate the cylinder kernel directly from the spectral decomposition as

$$
T(t, x, y)=\frac{2}{L} \sum_{n=1}^{\infty} \sin \left(\frac{n \pi x}{L}\right) \sin \left(\frac{n \pi y}{L}\right) e^{-(n \pi / L)^{2} t}
$$

or by the method of images as a sum over classical paths,

$$
T(t, x, y)=\frac{t}{\pi} \sum_{N=-\infty}^{\infty}\left[\frac{1}{(x-y-2 N L)^{2}+t^{2}}-\frac{1}{(x+y-2 N L)^{2}+t^{2}}\right] .
$$

Either sum can be evaluated in closed form as

$$
T(t, x, y)=\frac{1}{2 L}\left[\frac{\sinh (\pi t / L)}{\cosh (\pi t / L)-\cos (\pi(x-y) / L)}-\frac{\sinh (\pi t / L)}{\cosh (\pi t / L)-\cos (\pi(x+y) / L)}\right] .
$$

It follows that

$$
\operatorname{Tr} T=\frac{1}{2} \frac{\sinh (\pi t / L)}{\cosh (\pi t / L)-1}-\frac{1}{2} \sim \frac{L}{\pi t}-\frac{1}{2}+\frac{\pi t}{12 L}+O\left(t^{3}\right)
$$

Thus the energy, the $O(t)$ term times $-\frac{1}{2}$, is

$$
E=-\frac{\pi}{24 L}
$$

This formula has been known for many years (e.g., [5]).

Another simple example, or class of them, involves a vector bundle over the circle, coordinatized as $\Omega=(0, L)[42,28]$. Again $H=-d^{2} / d x^{2}$, but now the field is "twisted" so that

$$
u(L)=e^{i \theta} u(0), \quad u^{\prime}(L)=e^{i \theta} u^{\prime}(0) .
$$

The eigenfunctions and eigenvalues are

$$
\varphi_{n}(x)=e^{i(2 \pi n+\theta) x / L)} \quad(n \in \mathbf{Z}), \quad \omega_{j}^{ \pm}=\frac{2 \pi j \pm \theta}{L} \quad\left(j \in \mathbf{N} \text { or } \mathbf{Z}^{+}\right) .
$$


One can then show (see Section 3) that

$$
E_{\theta}=-\frac{\pi}{L} B_{2}\left(\frac{\theta}{2 \pi}\right)=-\frac{\pi}{12 L}\left[2-6 \frac{\theta}{\pi}+3\left(\frac{\theta}{\pi}\right)^{2}\right] .
$$

( $B_{2}$ is a Bernoulli polynomial.) What is intriguing about this example is its dependence on the parameter $\theta ; E_{\theta}$ can be positive as well as negative. Its extreme values are $E_{0}=-\frac{\pi}{6 L}$ and $E_{\pi}=+\frac{\pi}{12 L}$; these are the cases where the two eigenvalue sequences coincide, so the gaps in the spectrum are largest. $E_{\theta}$ passes through 0 for $\theta \approx 0.42$, which is close to the point $\pi / 2$ where the eigenvalues are equally spaced. We observe that for an individual eigenvalue sequence, the sign and magnitude of its contribution to the energy are determined by the phase of the spectral oscillation. Here that phase is controlled by $\theta$; in higher-dimensional systems the phase of the oscillations associated with a periodic classical orbit is controlled by the famous [KramersMorse-Keller-Gutzwiller-]Maslov index (see, e.g., [47]), whose significance for vacuum energy has only begun to be explored [55, 56, 23].

\subsection{Vacuum energy density}

For present purposes the energy density can be defined simply by leaving out the integration over $x$ in the cylinder trace and proceeding as before. Let $P(\lambda, x, y)$ be the integral kernel of the orthogonal projection onto the part of $L^{2}(\Omega)$ corresponding to spectrum less than or equal to $\lambda$; since

$$
K(t, x, y)=\int_{0}^{\infty} e^{-t \lambda} d P(\lambda, x, y)
$$

we can also define $P$ as the inverse Laplace transform of the heat kernel (the exact heat kernel, not its small- $t$ series). Then

$$
T(t, x, y)=\int_{0}^{\infty} e^{-t \sqrt{\lambda}} d P(\lambda, x, y)
$$

and, as mentioned in Theorem 1, its trace has an expansion completely analogous to (2.2) with $x$-dependent coefficients:

$$
T(t, x, x) \sim \sum_{s=0}^{\infty} e_{s}(x) t^{-d+s}+\sum_{\substack{s=d+1 \\ s-d \text { odd }}}^{\infty} f_{s}(x) t^{-d+s} \ln t .
$$

We can now define the energy density as

$$
E(x)=-\frac{1}{2} e_{1+d}(x) .
$$

In the quantum field theory, $E(x)$ (known in that context as $T_{00}(x)$ defined with $\xi=\frac{1}{4}$ ) is formally the finite part of

$$
\frac{1}{2}\left[\left(\frac{\partial u}{\partial t}\right)^{2}+u H u\right] .
$$

When the spectrum is discrete,

$$
P(\lambda, x, y)=\sum_{\lambda_{n} \leq \lambda} \varphi_{n}(x) \overline{\varphi_{n}(y)}
$$


and the integral of $P(\lambda, x, x)$ over $\Omega$ equals $N(\lambda)$, the number of eigenvalues less than or equal to $\lambda$. But one reason for the importance of the energy density is that it remains meaningful when $H$ has some continuous spectrum. If the spectrum is absolutely continuous,

$$
\sigma(\omega, x) \equiv \frac{d}{d \omega} P\left(\omega^{2}, x, x\right)
$$

exists (as a Radon-Nikodym derivative) and constitutes a local spectral density. (It is a density in two senses - with respect to both $\omega$ and $x$.)

Here, also, there is a prototypical example, the half-line with a Dirichlet endpoint:

$$
\Omega=(0, \infty), \quad H=-\frac{d^{2}}{d x^{2}}, \quad u(0)=0 .
$$

The eigenfunction expansion is the Fourier sine transform, equivalent to the projector

$$
P(\lambda, x, y)=\int_{0}^{\sqrt{\lambda}} \frac{2}{\pi} \sin (k x) \sin (k y) d k
$$

The solution for the cylinder kernel by the method of images is now a simpler analogue of (2.5):

$$
T(t, x, y)=\frac{t}{\pi}\left[\frac{1}{(x-y)^{2}+t^{2}}-\frac{1}{(x+y)^{2}+t^{2}}\right] .
$$

Thus

$$
T(t, x, x) \sim \frac{1}{\pi t}-\frac{t}{\pi(2 x)^{2}} \sum_{k=0}^{\infty}(-1)^{k}\left(\frac{t}{2 x}\right)^{2 k} \quad \text { as } t \downarrow 0,
$$

so

$$
E(x)=\frac{1}{8 \pi x^{2}} .
$$

Recall that in any one-dimensional billiard the untraced diagonal value of the heat kernel is simply

$$
K(t, x, x) \sim(4 \pi t)^{-d / 2}+O\left(t^{\infty}\right)
$$

(for fixed $x$ in the interior of $\Omega$ ) regardless of boundary conditions. In contrast, we see from (2.6), (2.10), and (2.13) that $T(t, x, x)$ is sensitive to the global geometry - the length of the interval, the nature of the boundary conditions, the structure (angle $\theta$ ) of the vector bundle. (In case (2.10), $E(x)$ is independent of $x$ and equals $E / L$.) Therefore, $T(t, x, x), E(x)$, and $E$ are interesting objects to study from the point of view of inverse problems and other aspects of spectral geometry.

For the finite interval (2.4), one finds

$$
E(x)=-\frac{\pi}{24 L^{2}}+\frac{\pi}{8 L^{2}} \csc ^{2}\left(\frac{\pi x}{L}\right) .
$$

This function is graphed in Fig. 1. Now

$$
\frac{\pi}{8 L^{2}} \csc ^{2}\left(\frac{\pi x}{L}\right) \sim \frac{1}{8 \pi x^{2}} \text { as } x \rightarrow 0,
$$

with a similar expansion as $x \rightarrow L$. Therefore, $E(x)$ displays the boundary behavior (2.14) in addition to a spatially homogeneous term. But now notice something strange: The "total" 


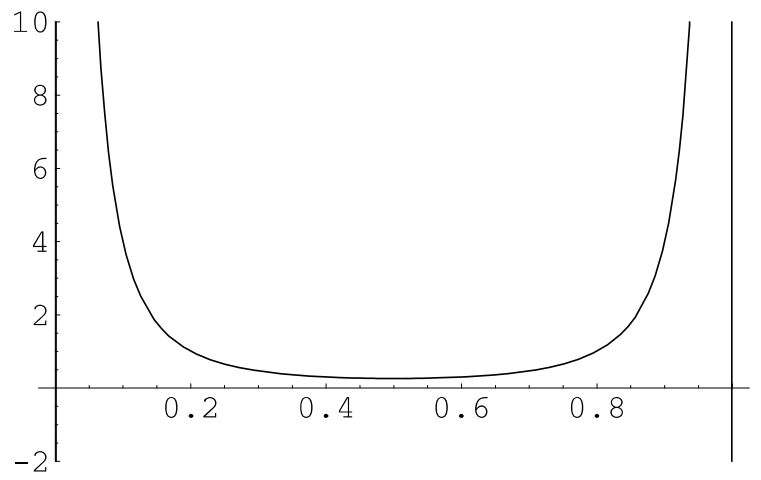

Figure 1. Boundary energy density for $\Omega=(0,1)$.

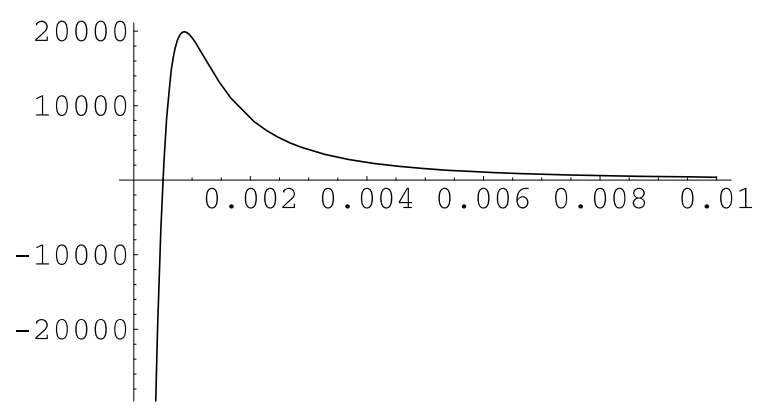

Figure 2. Regularized energy density $E(t, x)$ for $\Omega=(0, \infty)$ and $t=0.001$.

energy $E,(2.7)$, is equal to the integral over $\Omega$ of the spatially homogeneous term only, while the integral of the boundary terms diverges! In physical terms, the renormalized energy is not equal to the integral of the renormalized energy density. The physical significance of this fact is beyond the scope of this article. (In fact, it is still controversial.) Mathematically, however, it is just an instance of nonuniform convergence: The limit $t \rightarrow 0$ cannot be interchanged with the limit $x \rightarrow 0$, and hence with the integration over $x$.

To examine this phenomenon more closely, it is convenient to return to the half-line (2.11). Since the latter is spatially infinite, one would not necessarily expect its total energy to be defined; however, we notice that the integral of (2.14) converges at infinity. Let us keep $t$ positive (but small) and consider the regularized vacuum energy density

$$
E(t, x)=-\frac{1}{2} \frac{\partial}{\partial t} T(t, x, x)-\frac{1}{\pi t^{2}}=-\frac{1}{2 \pi} \frac{t^{2}-4 x^{2}}{\left(t^{2}+4 x^{2}\right)^{2}} .
$$

(See Fig. 2, noting the compressed vertical scale. The subtracted term is the contribution of the first term in (2.12) (or the first term in (2.13)), identified with the ubiquitous but unobservable vacuum energy of infinite empty space. Only the second term in (2.12) is of interest.) The limit $t \rightarrow 0$ reproduces the renormalized energy density (2.14), which would imply an infinite total energy if integrated. However,

$$
E(t) \equiv \int_{0}^{\infty} E(t, x) d x=0 \quad \text { for all } t>0,
$$

which implies a vanishing total energy if one now takes $t \rightarrow 0$. This disappearing divergence is a general property of energy densities that behave like $x^{-2}$ near a boundary. (It has been confirmed for the corners of a rectangle or rectangular parallelepiped [23].) By dimensional analysis any correlate of such a term in $E(t)$ must be proportional to $t^{-1}$ and hence must come from a term $f_{0} \ln t$ in (2.2), but no such term can exist. (It would necessarily match a $\ln t$ term in the heat kernel $[14,33,6,26]$, which does not exist for the class of operators considered here.) For the finite interval, it will be shown in Section 4 that the regularized total energy does not vanish, but does approach the renormalized energy (2.7) as $t \rightarrow 0$.

\subsection{But what about the zeta function?}

Many mathematically inclined researchers on vacuum energy have defined it in terms of zeta functions. For example, (2.7) is often expressed as

$$
\frac{1}{2} \sum_{n=1}^{\infty} \frac{n \pi}{L} "=" \frac{\pi}{2 L} \zeta(-1)=-\frac{\pi}{24 L},
$$


where $\zeta$ is the original Riemann zeta function. Therefore, a comment is needed upon the relation of generalized zeta functions to the approach expounded here.

Let $s$ be a complex parameter, and consider the operator function $f_{s}(H) \equiv H^{-s}$. Then the zeta function for $H$ is defined by $\zeta(s, H) \equiv \operatorname{Tr} f_{s}(H)$ and extended by analytic continuation to values of $s$ for which

$$
\zeta(s, H)=\sum_{n=1}^{\infty} \lambda^{-s}
$$

does not converge. Note that

$$
\zeta(s, H)=\zeta(2 s, \sqrt{H}) .
$$

On the other hand, these zeta functions are related to our integral kernels by

$$
\int_{0}^{\infty} t^{s-1} T(t, H) d t=\Gamma(s) \zeta(s, \sqrt{H})
$$

and a similar equation with $K(t, H)$. It follows from (2.1) and (2.2) that $b_{n}$ and $e_{n}$ are residues at poles of $\Gamma(s) \zeta(s, H)\left(\right.$ at $\left.s=\frac{1}{2}(d-n)\right)$ and $\Gamma(s) \zeta(s, \sqrt{H})($ at $s=d-n)$, respectively. So when there are no logarithmic terms, (2.16) implies

$$
\Gamma\left(\frac{d-n}{2}\right)^{-1} b_{n}=\frac{1}{2} \Gamma(d-n)^{-1} e_{n} .
$$

(This observation is analogous to one by Gilkey [31] concerning higher-order operators.) Now when $d-n$ is odd and negative, $\Gamma(d-n)$ has a pole where $\Gamma\left(\frac{1}{2}(d-n)\right)$ does not; the information in the corresponding $e_{n}$ is thereby expunged from the heat-kernel expansion, and that is how (2.1) contains less information than (2.2). In that case, $e_{n}$ ceases to be a residue of the zeta function and becomes a value of zeta at a regular point - a more subtle object to calculate. (Logarithmic terms give rise to coinciding poles of $\zeta$ and $\Gamma$.)

\section{Vacuum energy in a vector bundle from periodic orbits}

Let us concentrate now on the model studied in [28] and summarized above. $H=-\frac{d^{2}}{d x^{2}}$ acts in $L^{2}(0, L)$ with the modified periodic boundary conditions $(2.8)$, representing a nontrivial holonomy in a line bundle over the circle. For definiteness take $\theta \in[0,2 \pi)$. (A gauge transformation, $\tilde{\varphi}(x) \equiv e^{i \theta x / L} \varphi(x)$, converts this problem to "Bohm-Aharonov" form with ordinary periodic boundary conditions and a nonzero, but pure gauge, vector potential.) Eigenfunctions must be proportional to $e^{i k_{j} x}$ with $k_{j}=(2 \pi j+\theta) / L, j \in \mathbf{Z}$; the eigenvalues for positive and negative $k$ then form the two sequences (2.9).

Because of the spatial homogeneity of the model, there is no distinction, except a factor $L$, between total energy and local energy density. Although vacuum energy is barely mentioned in [28], (2.10) is equivalent to either of the two main results of that paper, the cylinder kernel for $H$ and its small- $t$ diagonal expansion [28, (9) and (13)], and the first nonlocal Riesz mean of the eigenvalue density with respect to frequency $\omega[28$, equation above (30)]. Here I rederive (2.10) in a third way, in the framework of periodic-orbit theory $[1,15,19]$ (but using the exact formula for the Green function, not a stationary-phase approximation).

The Green function (resolvent kernel) for the time-independent Schrödinger equation in infinite one-dimensional space is

$$
G_{\infty}\left(\omega^{2}, x, y\right)=\frac{i}{2 \omega} e^{i \omega|x-y|}
$$


(By convention, for $\omega^{2}$ on the positive real axis $G_{\omega}$ is defined as the limit from above $(\omega \mapsto$ $\omega+i \epsilon)$, or, equivalently, by the outgoing radiation condition. Also, let us adopt the convention $\left(H_{x}-\lambda\right) G(\lambda, x, y)=+\delta(x-y)$, where much of the physical literature has the opposite sign.) The Green function for the bundle is constructed as a sum over all classical paths connecting $y$ to $x$ in the covering space; in this simple one-dimensional problem this construction reduces to the traditional "method of images". When $y=x$ the paths become periodic orbits, and there is one for each $n \in \mathbf{Z}$, with length $|n| L$. Since there are no boundaries to reflect the paths, in this model there is no distinction between periodic orbits and more general closed paths (with different initial and final velocity), in keeping with the previous observation that energy and energy density are the same thing. The Green function $G=G_{L, \theta}$ is

$$
G\left(\omega^{2}, x, y\right)=\sum_{n=-\infty}^{\infty} G_{\infty}\left(\omega^{2}, x, y+n L\right) e^{i n \theta}
$$

(The sum converges distributionally and has the desired twisted-periodicity property.)

The starting point of periodic-orbit theory is that the density of eigenvalues, as a function of $\lambda$, is

$$
\sum_{j} \delta\left(\lambda-\lambda_{j}\right)=\frac{1}{\pi} \operatorname{Im} \operatorname{Tr} G(\lambda) .
$$

The trace is an integration over $x$, amounting here to a factor $L$. It is more convenient to work either with the density with respect to $\omega=\sqrt{\lambda}$, which carries an additional factor $2 \omega$, or with the eigenvalue counting function $N$, which is the same quantity whether $\lambda$ or $\omega$ is used as independent variable. We have

$$
\begin{aligned}
\operatorname{Im} G\left(\omega^{2}, x, x\right) & =\operatorname{Im} \sum_{n=-\infty}^{\infty} \frac{i}{2 \omega} e^{i \omega|n| L} e^{i n \theta} \\
& =\frac{1}{2 \omega}+\frac{1}{2 \omega} \sum_{n=1}^{\infty}[\cos (\omega n L+n \theta)+\cos (\omega n L-n \theta)] .
\end{aligned}
$$

(One can identify $\omega|n| L$ as the action of the $n$th periodic orbit. There is no Maslov index in this problem, but there is a phase shift $n \theta$ from the nontrivial holonomy.) So the integrated eigenvalue density is (for $\omega>0$ )

$$
\begin{aligned}
N(\omega) & =\frac{L}{\pi} \int 2 \omega d \omega \operatorname{Im} G\left(\omega^{2}, x, x\right)=\frac{L \omega}{\pi}+\frac{1}{\pi} \sum_{n=1}^{\infty} \frac{1}{n}[\sin (\omega n L+n \theta)+\sin (\omega n L-n \theta)] \\
& \equiv N_{\mathrm{av}}(\omega)+N_{\mathrm{osc}}(\omega) .
\end{aligned}
$$

Here $N_{\mathrm{av}}(\omega) / L=\omega / \pi$ (which came from the orbit of zero length) is the density of states per unit length that would exist in infinite space, and $N_{\text {osc }}(\omega)$ describes the bunching of spectrum caused by the existence of closed orbits. (The contribution of orbit $n$ to (3.1) includes an $\omega$ independent constant of integration, $-\sin (n \theta) / n \pi$, which cancels with the corresponding term from orbit $-n$.)

Before turning to the vacuum energy, let's digress to see how (3.1) reproduces the known eigenvalues. It is known $[34,1.441 .1,9.627 .1]$ that for $0<z<2 \pi$

$$
\sum_{n=1}^{\infty} \frac{\sin n z}{n}=\pi-\frac{z}{2}=-\pi B_{1}\left(\frac{z}{2 \pi}\right),
$$


where $B_{1}$ is the first Bernoulli polynomial; elsewhere, the sum defines a $2 \pi$-periodic sawtooth function, vanishing at the discontinuity points $z=2 j \pi$. Thus, for $\omega>0$,

$$
N_{\mathrm{osc}}(\omega)=\frac{1}{2 \pi}[f(\pi-\omega L-\theta)+f(\pi-\omega L+\theta)]
$$

where $f(\zeta)$ is the $2 \pi$-periodic extension of the function equal to $\zeta$ when $-\pi<\zeta<\pi$ and equal to 0 at the endpoints. Hence $f(\zeta)$ jumps by $-2 \pi$ at each odd multiple of $\pi$. In more transparent terms, the eigenvalue density is (since $N_{\text {av }}$ and $N_{\text {osc }}$ vanish for $\omega \leq 0$ )

$$
\rho(\omega) \equiv \frac{d N}{d \omega}=\frac{L}{\pi}+N_{\mathrm{osc}}^{\prime}(\omega)=\sum_{j=1}^{\infty} \delta\left(\omega-\frac{2 j \pi-\theta}{L}\right)+\sum_{j=0}^{\infty} \delta\left(\omega-\frac{2 j \pi+\theta}{L}\right),
$$

in precise agreement with the eigenvalues calculated already by elementary means. Of course, in higher-dimensional problems such exact results are not to be expected.

The renormalized vacuum energy is the contribution of $N_{\text {osc }}^{\prime}$ to $\int_{0}^{\infty} \frac{1}{2} \omega \rho(\omega) d \omega$. Returning to (3.1), and considering the local energy density, we have (ignoring analytical technicalities for the moment)

$$
E(x)=\int_{0}^{\infty} \frac{1}{2 L} \omega N_{\mathrm{osc}}^{\prime}(\omega) d \omega=\frac{1}{2 \pi} \sum_{n=1}^{\infty} \int_{0}^{\infty}[\cos (\omega n L+n \theta)+\cos (\omega n L-n \theta)] \omega d \omega .
$$

Consider just one term, and integrate to a finite upper limit:

$$
\int_{0}^{\Omega} \cos (\omega n L+n \theta) \omega d \omega=\frac{\Omega}{n L} \sin (\Omega n L+n \theta)+\frac{1}{(n L)^{2}} \cos (\Omega n L+n \theta)-\frac{1}{(n L)^{2}} \cos (n \theta) .
$$

As $\Omega \rightarrow \infty$, the first two terms oscillate with zero mean. If we assume for the moment that they can be ignored, we have

$$
-\frac{1}{2 \pi(n L)^{2}} \cos (n \theta)
$$

as the contribution to the energy density from one of the two periodic orbits of length $n L$; that from the other orbit comes out the same.

Again from [34, 1.443.3, 9.627.2], for $0<\theta<2 \pi$

$$
\sum_{n=1}^{\infty} \frac{\cos n \theta}{n^{2}}=\frac{\pi^{2}}{6}-\frac{\pi \theta}{2}+\frac{\theta^{2}}{4}=\pi^{2} B_{2}\left(\frac{\theta}{2 \pi}\right),
$$

where $B_{2}$ is the second Bernoulli polynomial. The total energy density is thus

$$
E(x)=-\frac{\pi}{L^{2}} B_{2}\left(\frac{\theta}{2 \pi}\right)=\frac{E_{\theta}}{L},
$$

$E_{\theta}$ given by (2.10), as was to be verified.

It is noteworthy that the vacuum energy (3.2) associated with a single spectral oscillation depends critically, in sign as well as magnitude, on the phase $n \theta$ of the oscillation. Algebraically, (3.2) comes entirely from the lower limit of the integration over $\omega$, and an effective lower cutoff on that integration would appear to change the phase. This is a matter of great concern for the extendability of the theory, since in more general circumstances the Gutzwiller spectral oscillations arise from stationary-phase approximations that are not justified at low frequency. It has often been observed, however, that periodic-orbit calculations reproduce low-lying eigenvalues more accurately than they have any right to do. 
The problem at high frequency, in contrast, is more apparent than real. The integral over $\omega$ can be defined by Riesz-Cesàro summation of order 2: A lengthy exercise in integration by parts shows that

$$
\int_{0}^{\Omega}\left(1-\frac{\omega}{\Omega}\right)^{2} \cos (\omega n L+n \theta) \omega d \omega=-\frac{1}{(n L)^{2}} \cos (n \theta)+O\left(\Omega^{-1}\right) .
$$

Taking the limit $\Omega \rightarrow \infty$ now yields (3.2). Clearly, the interchange of integration and summation in the calculation of $E(x)$ is now also justifiable.

Another approach leading to the same conclusion is Abel summation, which amounts to finding the contribution of each spectral oscillation to the cylinder kernel. We are after the small- $t$ behavior of

$$
\int_{0}^{\infty} \frac{1}{2} \omega \rho(\omega) e^{-\omega t} d \omega=-\frac{1}{2} \frac{d}{d t} \int_{0}^{\infty} \rho(\omega) e^{-\omega t} d \omega
$$

Using the integral

$$
\int_{0}^{\infty} \cos (a \omega-b) e^{-\omega t} d \omega=\frac{t}{t^{2}+a^{2}} \cos b+\frac{a}{t^{2}+a^{2}} \sin b,
$$

one finds that the contribution of the two orbits with length $n L$ to $\int_{0}^{\infty} \rho(\omega) e^{-\omega t} d \omega$ is

$$
\frac{2 L}{\pi} \frac{t}{t^{2}+(n L)^{2}} \cos (n \theta)
$$

(The two contributions are not equal; their sine terms cancel. But because the sine term in (3.3) has no term of order exactly $t^{+1}$ in its small- $t$ expansion, it would make no contribution to (3.2) even before the pairwise cancellation.) The sum over $n$ is now absolutely convergent, and at $t=0$ the expected formulas (3.2) and (2.10) emerge. Alternatively, the series at finite $t$ can be summed in closed form [34, 1.445.2], and with the help of some identities for hyperbolic functions the result is shown equal to the diagonal value of the cylinder kernel as found previously [28, $(12)]$.

If $\theta$ is written $|\theta|,(2.10)$ is valid over the interval $-2 \pi \leq \theta \leq 2 \pi$. Overall, $E_{\theta}$ is a continuous $2 \pi$-periodic function with cusps at the integer multiples of $2 \pi$; it is symmetric under reflection about any integer multiple of $\pi$. We have already commented in Section 2 about the significance of its extreme values and its zero.

\section{Boundary vacuum energy from closed and periodic orbits}

We consider a finite interval with either a Dirichlet or a Neumann boundary condition at each end. Thus $H=-\frac{d^{2}}{d x^{2}}$ acts in $L^{2}(0, L)$ on the domain defined by

$$
u^{(1-l)}(0)=0, \quad u^{(1-r)}(L)=0, \quad \text { where } l, r \in\{0,1\} .
$$

For $l=1$, the (unnormalized) eigenfunctions are $\sin \left(\omega_{j} x\right)$ with

$$
\omega_{j}=\frac{\pi j}{L}, \quad j \in \mathbf{Z}^{+}, \quad \text { if } r=1 ; \quad \omega_{j}=\frac{\pi}{L}\left(j+\frac{1}{2}\right), \quad j \in \mathbf{N}, \quad \text { if } r=0 .
$$

For $l=0$, they are $\cos \left(\omega_{j} x\right)$ with $j \in \mathbf{N}$ and

$$
\omega_{j}= \begin{cases}\frac{\pi}{L}\left(j+\frac{1}{2}\right) & \text { if } r=1, \\ \frac{\pi j}{L} & \text { if } r=0 .\end{cases}
$$


The Green function can be constructed from $G_{\infty}$ by the method of images, or, equivalently, as a sum over reflected paths as in [57, Fig. 1]. (I suppress the arguments $\omega^{2}$ and $x$ after their first appearance.)

$$
\begin{aligned}
G\left(\omega^{2}, x, y\right)= & G_{\infty}(y)+(-1)^{l} G_{\infty}(-y)+(-1)^{r} G_{\infty}(2 L-y)+(-1)^{l+r} G_{\infty}(2 L+y) \\
& +(-1)^{l+r} G_{\infty}(-2 L+y)+(-1)^{2 l+r} G_{\infty}(-2 L-y) \\
& +(-1)^{l+2 r} G_{\infty}(4 L-y)+(-1)^{2 l+2 r} G_{\infty}(4 L+y)+\cdots \\
= & \sum_{n=0}^{\infty}(-1)^{n(l+r)} G_{\infty}(-2 n L+y)+\sum_{n=0}^{\infty}(-1)^{l+n(l+r)} G_{\infty}(-2 n L-y) \\
& +\sum_{n=1}^{\infty}(-1)^{-l+n(l+r)} G_{\infty}(2 n L-y)+\sum_{n=1}^{\infty}(-1)^{n(l+r)} G_{\infty}(2 n L+y) \\
= & \frac{i}{2 \omega} \sum_{n=0}^{\infty}(-1)^{n(l+r)} e^{i \omega|2 n L+x-y|}+\frac{i}{2 \omega} \sum_{n=0}^{\infty}(-1)^{l+n(l+r)} e^{i \omega(2 n L+x+y)} \\
& +\frac{i}{2 \omega} \sum_{n=1}^{\infty}(-1)^{-l+n(l+r)} e^{i \omega(2 n L-x-y)}+\frac{i}{2 \omega} \sum_{n=1}^{\infty}(-1)^{n(l+r)} e^{i \omega(2 n L-x+y)}
\end{aligned}
$$

(cf. $[57,(2.16)]$ ). When $y=x$, the first and fourth sums correspond to periodic paths (including the direct path of zero length), but the second and third sums come from paths that are closed but not periodic (i.e., the "particle" has hit the boundary an odd number of times and returned to its starting point with reversed velocity). The $n=0$ term in the first sum is the direct path; $n=0$ in the second term and $n=1$ in the third (which will become $n=-1$ in the next step) are the "short" paths that bounce off the boundary only once.

We have (always with the understanding that $\omega>0$ )

$$
\begin{aligned}
\pi \sigma \equiv & 2 \omega \operatorname{Im} G\left(\omega^{2}, x, x\right) \\
= & \sum_{n=0}^{\infty}(-1)^{n(l+r)} \cos (2 \omega n L)+\sum_{n=0}^{\infty}(-1)^{l+n(l+r)} \cos (2 \omega(n L+x)) \\
& +\sum_{n=1}^{\infty}(-1)^{-l+n(l+r)} \cos (2 \omega(n L-x))+\sum_{n=1}^{\infty}(-1)^{n(l+r)} \cos (2 \omega n L) \\
= & 1+2 \sum_{n=1}^{\infty}(-1)^{n(l+r)} \cos (2 \omega n L)+\sum_{n=-\infty}^{\infty}(-1)^{l+n(l+r)} \cos (2 \omega(x+n L)) \\
\equiv & \pi\left(\sigma_{\mathrm{av}}+\sigma_{\mathrm{per}}+\sigma_{\mathrm{bdry}}\right) \equiv \pi\left(\sigma_{\mathrm{av}}+\sigma_{\mathrm{osc}}\right) .
\end{aligned}
$$

Because the series are not absolutely convergent, we must be wary of cancelling contributions from different values of $n$. For the most part, we want to consider to the latest possible moment the contribution from each orbit separately, and the summation is just a reminder of which values of $n$ occur. We do pair $n$ with $-n$ or $-(n+1)$ when their contributions are manifestly identical or parallel. We have just done so for the first and fourth terms in $\sigma$, which became $\sigma_{\mathrm{av}}+\sigma_{\mathrm{per}}$. In the second and third terms, $\sigma_{\mathrm{bdry}}$, it will be natural to pair $n(>0)$ with $-(n+1)$, since that combines paths that have the same number of reflections, $2 n+1$ (again consult [57, Fig. 1], or just look at the exponents of $(-1)$ in the formula). At fixed $x$, orbits $n$ and $-(n+1)$ do not have the same length, but when integrated over $x$ those two sets of orbits collectively occupy the same part of the length spectrum.

Let us compare with the case of a single boundary (Hilbert space $L^{2}(0, \infty)$, boundary condition $\left.u^{(1-l)}(0)=0\right)$. In that case only the direct path and the short path off the boundary exist. Thus $\sigma_{\mathrm{per}}=0, \sigma_{\mathrm{av}}$ is unchanged, and $\sigma_{\mathrm{bdry}}=\frac{(-1)^{l}}{\pi} \cos (2 \omega x)$ has only one term. 
There are now two routes to follow: We can work with the local spectral density $\sigma$ (integrating with respect to $\omega$, summing over $n$, calculating the local energy density, etc.), or we can integrate over $x$ first to get the global eigenvalue density. (Unfortunately, "density" is ambiguous in this context, as previously remarked.)

Let's examine the global situation first. The eigenvalue density is

$$
\rho(\omega)=\int_{0}^{L} \sigma(\omega, x) d x=\rho_{\mathrm{Weyl}}+\rho_{\mathrm{per}}+\rho_{\mathrm{bdry}}
$$

where

$$
\begin{aligned}
& \rho_{\text {Weyl }}=\int_{0}^{L} \sigma_{\text {av }} d x=\frac{L}{\pi}, \quad \rho_{\text {per }}=\frac{2 L}{\pi} \sum_{n=1}^{\infty}(-1)^{n(l+r)} \cos (2 \omega n L), \\
& \rho_{\text {bdry }}=\frac{(-1)^{l}}{2 \pi} \sum_{n=-\infty}^{\infty} \frac{(-1)^{n(l+r)}}{\omega}[\sin (2 \omega L(n+1))-\sin (2 \omega L n)] .
\end{aligned}
$$

In keeping with previous remarks, we refuse for the moment to take advantage of the possibility of "telescoping" $\rho_{\text {bdry }}$ when $l+r$ is even, but we can combine the positive and negative parts of the series now. In the summand $\frac{(-1)^{n(l+r)}}{\omega}[\sin (2 \omega L(n+1))-\sin (2 \omega L n)]$, replace $n$ by $-(n+1)$ :

$$
\begin{aligned}
& \frac{(-1)^{-(n+1)(l+r)}}{\omega}[\sin (2 \omega L(-n))-\sin (-2 \omega L(n+1))] \\
& =(-1)^{l+r} \frac{(-1)^{n(l+r)}}{\omega}[\sin (2 \omega L(n+1))-\sin (2 \omega L n)],
\end{aligned}
$$

which is the original summand except for the initial sign. Therefore, if $l+r$ is odd (the two boundary conditions are different), $\rho_{\text {bdry }}=0$; but if $l+r$ is even,

$$
\rho_{\text {bdry }}=\frac{(-1)^{l}}{\pi} \sum_{n=0}^{\infty} \frac{1}{\omega}[\sin (2 \omega L(n+1))-\sin (2 \omega L n)] .
$$

(The local counterpart of this observation is that the simultaneous transformation $n \mapsto-(n+1)$, $x \mapsto L-x$ leaves the summand invariant up to the sign. Together with the sign $(-1)^{l}$, this indicates that the effects that are localized near the boundaries are equal and opposite for Dirichlet and Neumann boundaries.)

The corresponding equation for the half-line with a single boundary is formally

$$
\rho_{\text {bdry }}=\frac{(-1)^{l}}{\pi} \int_{0}^{\infty} \cos (2 \omega x) d x=\frac{(-1)^{l}}{4} \delta(\omega) .
$$

Of course $\rho_{\mathrm{av}}$ is infinite in that case because of the infinite volume and continuous spectrum, but $\rho_{\text {bdry }}$, being associated with the boundary, is a localized, finite contribution that can be studied separately.

Remark. The distributional integral (4.1) has been thoroughly studied in [22]. The second equality in (4.1) is correct under the convention that

$$
f(0) \equiv \int_{0}^{\infty} \delta(\omega) f(\omega) d \omega=\int_{-\infty}^{\infty} \delta(\omega) H(\omega) f(\omega) d \omega
$$

where $H$ is the unit step function. An alternative convention is that a delta function at an endpoint of an interval of integration yields only half the value of the test function at that point; otherwise put, the integration is extended over all $\omega \in \mathbf{R}$ and the integrand is interpreted 
according to the rule $\delta(\omega) \theta(\omega)=\frac{1}{2} \delta(\omega)$. The choice is somewhat arbitrary [22], but (4.2) has the advantage that an eigenvalue $\omega_{0}^{2}$ always corresponds in $\rho$ to a unit-normalized delta function, $\delta\left(\omega-\omega_{0}\right)$, even when $\omega_{0}=0$.

In a more general problem, the integrals over $\omega$ would need to be evaluated in a stationaryphase approximation. It would then be argued that only periodic orbits contribute, so $\rho_{\text {bdry }}$ would be set to zero. A formal justification for this approximation is that the neglected terms are of higher order in Planck's constant when nonrelativistic quantum-mechanical units are used in $H$. In relativistic vacuum-energy calculations in natural units, the manifestation of this observation is that $\rho_{\text {bdry }}$ is suppressed relative to $\rho_{\text {av }}+\rho_{\text {per }}$ by a factor $1 / \omega L$, which is small in the high-frequency regime where WKB-type asymptotics would be valid. A less drastic approximation is to keep only the two short orbits:

$$
\rho_{\mathrm{bdry}} \approx \frac{(-1)^{l}}{\pi} \frac{\sin (2 \omega L)}{\omega}
$$

This is plausible because the boundary effect should come only from points close to the boundary (cf. the local calculations below and the single-boundary equation above).

The eigenvalue counting function $N(\omega)$ is zero for $\omega<0$ and $\int_{0}^{\omega} \rho$ for $\omega>0$. Therefore (for $\omega>0)$,

$$
\begin{aligned}
& N_{\text {Weyl }}=\frac{L \omega}{\pi}, \quad N_{\text {per }}=\frac{1}{\pi} \sum_{n=1}^{\infty} \frac{(-1)^{n(l+r)}}{n} \sin (2 \omega n L), \\
& N_{\text {bdry }}= \begin{cases}\frac{(-1)^{l}}{\pi} \sum_{n=0}^{\infty} \int_{0}^{\omega} \frac{\sin (2 \omega L(n+1))-\sin (2 \omega L n)}{\omega} d \omega & \text { if } l+r \text { is even, } \\
0 & \text { if } l+r \text { is odd. } .\end{cases}
\end{aligned}
$$

We contemplate each of these in turn.

$N_{\text {Weyl }}$ is exactly as expected.

If $l+r$ is odd, by $[34,1.441 .3]$ we have

$$
N_{\text {per }}=\frac{1}{\pi} \sum_{n=1}^{\infty} \frac{(-1)^{n}}{n} \sin (2 \omega n L)=-\frac{1}{2 \pi} f(2 \omega L)
$$

with the same $f(\zeta)$ as defined in Section 3. That is, if we temporarily forget the restriction to $\omega>0, N_{\text {per }}$ equals $-\frac{2 \omega L}{2 \pi}=-N_{\text {Weyl }}$ for $\omega \in\left(-\frac{\pi}{2 L}, \frac{\pi}{2 L}\right)$, vanishes at the endpoints, and is periodic thereafter; this function jumps upward by 1 at each (positive) odd multiple of $\frac{\pi}{2 L}$, which we know to be the correct eigenvalues for these problems. (Another way of saying this sort of thing is that $N_{\text {per }}$ is equal to the negative of $N_{\text {Weyl }}$, plus a series of unit step functions located at the eigenvalues.) At $\omega=0$ this function equals 0 , so the complete $N_{\text {per }}$ is continuous there. For consistency, $N_{\text {bdry }}$ must turn out to be 0 when $l+r$ is odd (and it does).

If $l+r$ is even,

$$
N_{\text {per }}=\frac{1}{\pi} \sum_{n=1}^{\infty} \frac{\sin (2 \omega n L)}{n}=\frac{1}{2 \pi} f(\pi-2 \omega L),
$$

so the jumps occur at the (positive) integer multiples of $\frac{\pi}{L}$, as they should. The only complication is at $\omega=0$, where this function approaches $\frac{1}{2}$ from the right. Since the complete $N_{\text {per }}$ is 0 for $\omega<0$, there is only half a step function at 0 . In the full $N$, there should be a complete unit jump in the Neumann problem $(l=0)$ and no jump at all in the Dirichlet problem $(l=1)$. These corrections must come, of course, from the boundary term. 
For it we have

$$
\begin{aligned}
N_{\text {bdry }} & =\frac{(-1)^{l}}{\pi} \sum_{n=0}^{\infty}\left[\int_{0}^{2 L \omega(n+1)} \frac{\sin u}{u} d u-\int_{0}^{2 L \omega n} \frac{\sin u}{u} d u\right] \\
& =\frac{(-1)^{l}}{\pi} \sum_{n=0}^{\infty} \int_{2 L \omega n}^{2 L \omega(n+1)} \frac{\sin u}{u} d u=\frac{(-1)^{l}}{\pi} \int_{0}^{\infty} \frac{\sin u}{u} d u=\frac{(-1)^{l}}{2}
\end{aligned}
$$

(see, e.g., [34, 3.721.1]). This holds for $\omega>0$; thus $N_{\text {bdry }}$ heals the discontinuity at $\omega=0$ in $N_{\text {per }}$ when $l=1$ and strengthens it to a unit jump when $l=0$, as expected.

Also noteworthy, although not unexpected, are that $N_{\text {bdry }}$ is independent of $L$ - it is associated with the physics of the boundary, not the finiteness of the region - and that it is nonoscillatory as a function of $\omega$. Thus for $N$ (and other global quantities) it seems proper to write

$$
N_{\mathrm{av}} \equiv N_{\text {Weyl }}+N_{\text {bdry }}, \quad N_{\text {osc }} \equiv N_{\text {per }},
$$

in contrast to the definition of $\sigma_{\mathrm{av}}$ and $\sigma_{\mathrm{osc}}$.

If we kept only the contribution from the short orbits, we would get

$$
N_{\mathrm{bdry}} \approx \frac{(-1)^{l}}{\pi} \int_{0}^{2 L \omega} \frac{\sin u}{u} d u
$$

a fair approximation to the correct step function when $L \omega$ is large. For comparison, our formula for $\rho_{\text {bdry }}$ on the half-line also yields a step function at the origin of half that magnitude, $\frac{(-1)^{l}}{4} H(\omega)$, as one should expect [10] for only a single boundary.

Now consider the regularized vacuum energy

$$
E(t)=-\frac{d}{d t} \frac{1}{2} \int_{0}^{\infty} \rho(\omega) e^{-\omega t} d \omega \equiv E_{\mathrm{Weyl}}+E_{\mathrm{per}}+E_{\mathrm{bdry}}
$$

where

$$
E_{\mathrm{Weyl}}(t)=-\frac{L}{2 \pi} \frac{d}{d t} \frac{1}{t}=\frac{L}{2 \pi t^{2}},
$$

and

$$
\begin{aligned}
E_{\mathrm{per}}(t) & =-\frac{L}{\pi} \frac{d}{d t} \sum_{n=1}^{\infty}(-1)^{n(l+r)} \int_{0}^{\infty} \cos (2 \omega n L) e^{-\omega t} d \omega \\
& =-\frac{L}{\pi} \frac{d}{d t} \sum_{n=1}^{\infty}(-1)^{n(l+r)} \frac{t}{t^{2}+(2 n L)^{2}}=-\frac{1}{2 \pi} \frac{d}{d t} \sum_{n=1}^{\infty}(-1)^{n(l+r)} \frac{t / 2 L}{(t / 2 L)^{2}+n^{2}} .
\end{aligned}
$$

These series can be evaluated by $[34,1.217 .1,2]$ or their generalizations $[34,1.445 .2,3]$ : In the even case,

$$
E_{\mathrm{per}}(t)=-\frac{1}{2 \pi} \frac{d}{d t}\left[\frac{\pi}{2} \operatorname{coth}\left(\frac{\pi t}{2 L}\right)-\frac{L}{t}\right]=\frac{\pi}{8 L} \operatorname{csch}^{2}\left(\frac{\pi t}{2 L}\right)-\frac{L}{2 \pi t^{2}} .
$$

In the odd case,

$$
E_{\mathrm{per}}(t)=-\frac{1}{2 \pi} \frac{d}{d t}\left[\frac{\pi}{2} \operatorname{csch}\left(\frac{\pi t}{2 L}\right)-\frac{L}{t}\right]=\frac{\pi}{8 L} \operatorname{csch}\left(\frac{\pi t}{2 L}\right) \operatorname{coth}\left(\frac{\pi t}{2 L}\right)-\frac{L}{2 \pi t^{2}} .
$$


Expand these in power series in $t$, using $[34,1.411 .8,12]$ :

$$
\operatorname{csch} z=\frac{1}{z}-\frac{z}{6}+O\left(z^{3}\right), \quad \operatorname{coth} z=\frac{1}{z}+\frac{z}{3}+O\left(z^{3}\right) .
$$

The terms of order $t^{-2}$ cancel, and we get

$$
E_{\mathrm{per}}(t)=\left\{\begin{array}{cl}
-\frac{\pi}{24 L}+O\left(t^{2}\right) & \text { if } l+r \text { is even } \\
\frac{\pi}{48 L}+O\left(t^{2}\right) & \text { if } l+r \text { is odd }
\end{array}\right.
$$

The first of these gives the well known renormalized vacuum energy (2.7) for the one-dimensional Dirichlet problem $(l=r=1)$. It is also correct for the Neumann case, when any energy associated with the indiscretely quantized zero mode is neglected. The extra factor of $-\frac{1}{2}$ in the mixed case is just like that for the antiperiodic case in Section 3 and [42] and similar to the factor of $-\frac{7}{8}$ in the mixed case in three-dimensional electromagnetism [8]; this family of formulas can be obtained at the eigenfunction level by doubling the interval and removing the contribution of the even modes [18].

It remains to investigate the boundary energy. As usual it will be zero in the odd case. For the even case we have

$$
\begin{aligned}
E_{\mathrm{bdry}}(t) & =-\frac{(-1)^{l}}{2 \pi} \frac{d}{d t} \sum_{n=0}^{\infty} \int_{0}^{\infty}[\sin (2 \omega L(n+1))-\sin (2 \omega L n)] \frac{e^{-\omega t}}{\omega} d \omega \\
& =\frac{(-1)^{l}}{2 \pi} \sum_{n=0}^{\infty} \int_{0}^{\infty}[\sin (2 \omega L(n+1))-\sin (2 \omega L n)] e^{-\omega t} d \omega \\
& =\frac{(-1)^{l}}{2 \pi} \sum_{n=0}^{\infty}\left[\frac{2 L(n+1)}{t^{2}+(2 L(n+1))^{2}}-\frac{2 L n}{t^{2}+(2 L n)^{2}}\right] .
\end{aligned}
$$

This series is conditionally convergent and telescopes to zero. Alternatively, put the two terms in the summand over a common denominator:

$$
E_{\mathrm{bdry}}(t)=\frac{(-1)^{l}}{2 \pi} \sum_{n=0}^{\infty} \frac{2 L t^{2}-(2 L)^{3} n(n+1)}{\left[t^{2}+(2 L(n+1))^{2}\right]\left[t^{2}+(2 L n)^{2}\right]} .
$$

The term for $n=0$ is

$$
\frac{(-1)^{l}}{\pi} \frac{L}{t^{2}+4 L^{2}}=\frac{(-1)^{l}}{4 \pi L}+O\left(t^{2}\right)
$$

In the other terms it is legitimate to expand the denominators before summing:

$$
\begin{aligned}
& \frac{(-1)^{l}}{2 \pi} \sum_{n=1}^{\infty} \frac{2 L t^{2}-(2 L)^{3} n(n+1)}{(2 L)^{4} n^{2}(n+1)^{2}}\left[1-\frac{t^{2}}{(2 L n)^{2}}+\cdots\right]\left[1-\frac{t^{2}}{(2 L(n+1))^{2}}+\cdots\right] \\
& \quad=-\frac{(-1)^{l}}{4 \pi L} \sum_{n=1}^{\infty} \frac{1}{n(n+1)}+O\left(t^{2}\right)=-\frac{(-1)^{l}}{4 \pi L}+O\left(t^{2}\right),
\end{aligned}
$$

the numerical sum being a textbook example [58, pp. 612-613] of a telescoping series that converges to 1 . So all these terms exactly cancel the $n=0$ term in the limit of small $t$, and $E_{\text {bdry }}(0)=0$. This result was not entirely obvious, since one might expect boundary energies (possibly infinite) at both ends with the same sign. 
In the approximation of keeping only the short orbits, one gets a nonzero (and $L$-dependent) result, $E_{\mathrm{bdry}}(0)=\frac{(-1)^{l}}{4 \pi L}$. For the half-line we have

$$
\begin{aligned}
E_{\mathrm{bdry}}(t) & =-\frac{1}{2} \frac{d}{d t} \int_{0}^{\infty} \frac{(-1)^{l}}{4} \delta(\omega) e^{-\omega t} d \omega \quad \text { or } \quad \frac{1}{2} \int_{0}^{\infty} \frac{(-1)^{l}}{4} \delta(\omega) \omega e^{-\omega t} d \omega \\
& =0
\end{aligned}
$$

Now we turn to local quantities. First, integrate $\sigma$ to get a local analogue of the counting function. (This is the inverse Laplace transform of the diagonal value of the heat kernel; it is the quantity called $\mu^{00}$ in [25].) As expected,

$$
\int \sigma_{\mathrm{av}} d \omega=\frac{\omega}{\pi}, \quad \int \sigma_{\mathrm{per}} d \omega=\frac{1}{\pi L} \sum_{n=1}^{\infty} \frac{(-1)^{n(l+r)}}{n} \sin (2 n L \omega)=\frac{1}{L} N_{\mathrm{per}} .
$$

The boundary term is

$$
\int \sigma_{\mathrm{bdry}} d \omega=\frac{1}{\pi} \sum_{n=-\infty}^{\infty}(-1)^{l+n(l+r)} \frac{\sin (2 \omega(x+n L)}{2(x+n L)} .
$$

Integration of it over $x$ yields the same $N_{\text {bdry }}$ found before. The analogous calculations for the half-line give

$$
\int \sigma_{\text {bdry }} d \omega=\frac{(-1)^{l}}{2 \pi} \frac{\sin (2 \omega x)}{x}
$$

which is precisely the $n=0$ term in the sum above, and

$$
N_{\text {bdry }}=\frac{(-1)^{l}}{2 \pi} \int_{0}^{\infty} \frac{\sin (2 \omega x)}{x} d x=\frac{(-1)^{l}}{4},
$$

in agreement with our previous result (4.1) for this case.

At this point let's pause to compare the results with those from a direct summation of the eigenfunction expansion, specializing to the doubly Dirichlet case, $l=r=1$. The diagonal value of the (integrated) spectral kernel is

$$
\sum_{(\pi j / L) \leq \omega}\left|\varphi_{j}(x)\right|^{2}=\sum_{j=1}^{\lfloor\omega L / \pi\rfloor} \frac{2}{L} \sin ^{2}\left(\frac{\pi j x}{L}\right),
$$

which reduces after some calculation to

$$
\frac{1}{2 L}+\frac{1}{L}\lfloor\omega L / \pi\rfloor-\frac{1}{2 L} \sum_{j=-\lfloor\omega L / \pi\rfloor}^{\lfloor\omega L / \pi\rfloor} e^{2 \pi i j x / L} .
$$

The first two terms are precisely the step function $\frac{1}{L}\left(N_{\mathrm{av}}+N_{\text {per }}\right)$. The sum in the final term is the Dirichlet kernel introduced in any rigorous textbook on Fourier series (e.g., [38, p. 22]). Thus

$$
\int \sigma_{\text {bdry }} d \omega=-\frac{1}{2 L} \frac{\sin \left(\left(1+2\left\lfloor\frac{\omega L}{\pi}\right\rfloor\right) \frac{\pi x}{L}\right)}{\sin \left(\frac{\pi x}{L}\right)} .
$$


When $\omega$ is large, this function develops sharp peaks near $x=0$ and $x=L$, in keeping with the boundary-effect picture we have had in mind all along. But instead of performing this sum, we can relate it to the closed-orbit calculation by the Poisson summation formula:

$$
\begin{aligned}
-2 L \int \sigma_{\text {bdry }} d \omega & =\sum_{j=-\lfloor\omega L / \pi\rfloor}^{\lfloor\omega L / \pi\rfloor} e^{2 \pi i j x / L}=\sum_{j=-\infty}^{\infty} e^{2 \pi i j x / L} \theta(\omega L-\pi|j|) \\
& =\sum_{n=-\infty}^{\infty} \int_{-\infty}^{\infty} d j e^{2 \pi i j n} e^{2 \pi i j x / L} \theta(\omega L-\pi|j|) \\
& =\sum_{n=-\infty}^{\infty} \int_{-\lfloor\omega L / \pi\rfloor}^{\lfloor\omega L / \pi\rfloor} e^{2 \pi i j(n+x / L)} d j=\sum_{n=-\infty}^{\infty} \frac{\sin (2 \omega(x+n L))}{x+n L} .
\end{aligned}
$$

Finally, we calculate the local energy density. The definition of the energy density of the massless scalar field in spatial dimension 1 in flat space is

$$
T_{00}(x)=\frac{1}{2}\left[\left(\frac{\partial \phi}{\partial t}\right)^{2}+\left(\frac{\partial \phi}{\partial x}\right)^{2}-4 \xi\left[\left(\frac{\partial \phi}{\partial x}\right)^{2}+\phi \frac{\partial^{2} \phi}{\partial x^{2}}\right]\right],
$$

where $\xi$ is called the conformal coupling parameter; different values of $\xi$ correspond to different theories of the coupling of the field to gravity, but in flat space they are physically and mathematically equivalent apart from this one definition. The term multiplied by $-4 \xi$ equals

$$
\frac{\partial}{\partial x}\left(\phi \frac{\partial \phi}{\partial x}\right)
$$

which classically vanishes upon integration by parts in free space or under either Dirichlet or pure Neumann boundary conditions. In dimension 1 there are only two distinguished values of $\xi$, namely 0 (the conformal, or Yamabe, choice) and $\frac{1}{4}$ (which is needed for energy conservation in the presence of general boundary conditions if singular surface terms are to be avoided [46, 53, $26])$. The energy density for general $\xi$ is a convex combination of these two special cases. In the conformal case in dimension 1 the contribution of each normal mode to the vacuum expectation value is independent of $x$, so we know that $T_{00}$ is just $E / L$. (In particular, there is no boundary contribution.) In the case $\xi=\frac{1}{4}$ the contribution of the space derivatives is identical to that of the time derivatives, so we can write

$$
T_{00}(t, x) \equiv E(t, x)=-\frac{1}{2} \frac{\partial}{\partial t} \int_{0}^{\infty} \sigma(\omega) e^{-\omega t} d \omega \equiv E_{\text {Weyl }}(t)+E_{\text {per }}(t)+E_{\text {bdry }}(t, x),
$$

which now is indeed the energy formula with the integration stripped off.

Clearly, for the first two terms we get the same old result,

$$
E_{\text {Weyl }}(0)+E_{\text {per }}(0)=\frac{E}{L} .
$$

The interesting term is

$$
\begin{aligned}
E_{\mathrm{bdry}}(t, x) & =-\frac{(-1)^{l}}{2 \pi} \frac{\partial}{\partial t} \sum_{n=-\infty}^{\infty}(-1)^{n(l+r)} \int_{0}^{\infty} \cos (2 \omega(x+n L)) e^{-\omega t} d \omega \\
& =-\frac{(-1)^{l}}{2 \pi} \frac{\partial}{\partial t} \sum_{n=-\infty}^{\infty}(-1)^{n(l+r)} \frac{t}{t^{2}+4(x+n L)^{2}} \\
& =\frac{(-1)^{l}}{2 \pi} \sum_{n=-\infty}^{\infty}(-1)^{n(l+r)} \frac{t^{2}-4(x+n L)^{2}}{\left[t^{2}+4(x+n L)^{2}\right]^{2}} .
\end{aligned}
$$


These sums do not appear in [34], but Mathematica [60] evaluates them in terms of hyperbolic functions of complex argument. Some hindsight reveals what is going on: The summations we used to evaluate the total energy (effectively the present sums with $x=0$ ) can be written

$$
\frac{\pi}{a} \operatorname{coth}(\pi a)=\sum_{n=-\infty}^{\infty} \frac{1}{n^{2}+a^{2}}, \quad \frac{\pi}{a} \operatorname{csch}(\pi a)=\sum_{n=-\infty}^{\infty} \frac{(-1)^{n}}{n^{2}+a^{2}} .
$$

Factoring the terms on the right, we see that these are just Mittag-Leffler expansions of the hyperbolic functions in terms of simple poles:

$$
2 \pi \operatorname{coth}(\pi a)=\sum_{n=-\infty}\left(\frac{1}{a+i n}+\frac{1}{a-i n}\right)
$$

and a similar formula for csch. So the summands with a general quadratic in the denominator can be treated by displacing the argument of the hyperbolic functions and letting $n \mapsto-n$ in half the terms:

$$
\begin{aligned}
& \sum_{n=-\infty}^{\infty} \frac{1}{(n+b)^{2}+a^{2}}=\frac{\pi}{2 a}[\operatorname{coth}(\pi(a+i b))+\operatorname{coth}(\pi(a-i b))], \\
& \sum_{n=-\infty}^{\infty} \frac{(-1)^{n}}{(n+b)^{2}+a^{2}}=\frac{\pi}{2 a}[\operatorname{csch}(\pi(a+i b))+\operatorname{csch}(\pi(a-i b))] .
\end{aligned}
$$

(These formulas are actually improvements on Mathematica's output, though equivalent.)

Thus when $l+r$ is even,

$$
E_{\mathrm{bdry}}(t, x)=-\frac{(-1)^{l}}{8 L} \frac{\partial}{\partial t}\left[\operatorname{coth}\left(\frac{\pi t}{2 L}+\frac{i \pi x}{L}\right)+\operatorname{coth}\left(\frac{\pi t}{2 L}-\frac{i \pi x}{L}\right)\right],
$$

and when $l+r$ is odd, the formula is the same with coth replaced by csch. Differentiating first, and then working out the complex arithmetic, one gets in the even case

$$
E_{\mathrm{bdry}}(t, x)=\frac{(-1)^{l} \pi}{8 L^{2}} \frac{\sinh ^{2}\left(\frac{\pi t}{2 L}\right) \cos ^{2}\left(\frac{\pi x}{L}\right)-\cosh ^{2}\left(\frac{\pi t}{2 L}\right) \sin ^{2}\left(\frac{\pi x}{L}\right)}{\left[\sinh ^{2}\left(\frac{\pi t}{2 L}\right) \cos ^{2}\left(\frac{\pi x}{L}\right)+\cosh ^{2}\left(\frac{\pi t}{2 L}\right) \sin ^{2}\left(\frac{\pi x}{L}\right)\right]^{2}}
$$

and in the odd case

$$
E_{\mathrm{bdry}}(t, x)=\frac{(-1)^{l} \pi}{8 L^{2}} \frac{\cosh \left(\frac{\pi t}{2 L}\right) \cos \left(\frac{\pi x}{L}\right)\left[\sinh ^{2}\left(\frac{\pi t}{2 L}\right)-\sin ^{2}\left(\frac{\pi x}{L}\right)+2 \sinh ^{2}\left(\frac{\pi t}{2 L}\right) \sin ^{2}\left(\frac{\pi x}{L}\right)\right]}{\left[\sinh ^{2}\left(\frac{\pi t}{2 L}\right) \cos ^{2}\left(\frac{\pi x}{L}\right)+\cosh ^{2}\left(\frac{\pi t}{2 L}\right) \sin ^{2}\left(\frac{\pi x}{L}\right)\right]^{2}} .
$$

Specializing to $l=1$, let us examine the leading terms at small $t$ and small $x$. For the even case,

$$
E_{\text {bdry }}(0, x)=\frac{\pi}{8 L^{2}} \csc ^{2}\left(\frac{\pi x}{L}\right) .
$$

This is the renormalized boundary energy density in (2.15) (Fig. 1). Its integral over the whole interval is not even finite, much less zero as formally expected. Near $x=0$ we have

$$
E_{\text {bdry }}(0, x)=\frac{1}{8 \pi x^{2}}+\frac{\pi}{24 L^{2}}+O\left(x^{2}\right)
$$

(and a corresponding expansion near $x=L$ ). On the other hand, if we expand in $x$ first we get

$$
E_{\text {bdry }}(t, x)=-\frac{\pi}{8 L^{2}} \operatorname{csch}^{2}\left(\frac{\pi t}{2 L}\right)+O\left(x^{2}\right),
$$


the remainder term being nonuniform in $t$. At $x=0$ and small $t$ this becomes

$$
E_{\mathrm{bdry}}(t, 0) \approx-\frac{1}{2 \pi t^{2}}
$$

- that is, infinitely negative! Plotting the exact $E_{\mathrm{bdry}}(t, x)$ for various small but nonzero values of $t$ reveals a steep rise as $x$ approaches the boundary, followed by an even steeper plunge to negative values still closer to the boundary (cf. Fig. 2). This behavior assures that $\int_{0}^{L} E_{\mathrm{bdry}}(t, x) d x=0$ for any nonzero $t$, so that the total energy (2.7) is independent of $\xi$, as it must be. (The integral has been evaluated directly in the half-line case (4.3), and with the cutoff in place there is no obstacle to integrating the original spectral sum term by term and observing that the totalderivative terms integrate to zero.) This mathematical phenomenon was pointed out by Ford and Svaiter [24].

The situation for the odd case is very similar. $E_{\mathrm{bdry}}(t, x)$ is now an odd function of $x-\frac{L}{2}$, with the same qualitative behavior as in the even case near $x=0$ and the inverted behavior at the other end of the interval. The formulas parallel to those above are

$$
\begin{aligned}
& E_{\mathrm{bdry}}(0, x)=\frac{\pi}{8 L^{2}} \cot \left(\frac{\pi x}{L}\right) \csc \left(\frac{\pi x}{L}\right), \\
& E_{\mathrm{bdry}}(0, x)=\frac{1}{8 \pi x^{2}}-\frac{\pi}{48 L^{2}}+O\left(x^{2}\right), \\
& E_{\mathrm{bdry}}(t, x)=-\frac{\pi}{8 L^{2}} \operatorname{coth}\left(\frac{\pi t}{2 L}\right) \operatorname{csch}\left(\frac{\pi t}{2 L}\right)+O\left(x^{2}\right), \\
& E_{\mathrm{bdry}}(t, 0) \approx-\frac{1}{2 \pi t^{2}} .
\end{aligned}
$$

For the problem with a single boundary at $x=0$ we have

$$
\begin{aligned}
E_{\text {bdry }}(t, x) & =-\frac{1}{2} \frac{\partial}{\partial t} \int_{0}^{\infty} \sigma_{\text {bdry }}(\omega, x) e^{-\omega t} d \omega=-\frac{1}{2} \frac{\partial}{\partial t} \int_{0}^{\infty} \frac{(-1)^{l}}{\pi} \cos (2 \omega x) e^{-\omega t} d \omega \\
& =-\frac{(-1)^{l}}{2 \pi} \frac{\partial}{\partial t} \frac{t}{t^{2}+4 x^{2}}=\frac{(-1)^{l}}{2 \pi} \frac{t^{2}-4 x^{2}}{\left(t^{2}+4 x^{2}\right)^{2}} .
\end{aligned}
$$

Restricted to $0<x<L$, this is exactly the $n=0$ term in the sum for the problem with two boundaries. (The other short path, $n=-1$, naturally gives a symmetrical contribution localized at the other boundary.) The leading terms are the same as found above for two boundaries. In particular,

$$
E_{\text {bdry }}(0, x)=\frac{(-1)^{l-1}}{8 \pi x^{2}}
$$

in agreement with the renormalized vacuum energy found by Romeo and Saharian [53, (3.21)]. At small $x$ and fixed $t$ we have

$$
E_{\text {bdry }}(t, x)=\frac{(-1)^{l}}{2 \pi}\left[\frac{1}{t^{2}}-\frac{12 x^{2}}{t^{4}}+O\left(\frac{x^{4}}{t^{6}}\right)\right],
$$

again giving some insight into the sharp spike of opposite sign that keeps the total boundary energy equal to zero as long as the regularization has not been removed. Indeed, in this case the integral of the exact function is elementary:

$$
\int_{0}^{\infty} \frac{t^{2}-4 x^{2}}{\left(t^{2}+4 x^{2}\right)^{2}} d x=\left.\frac{x}{t^{2}+4 x^{2}}\right|_{x=0} ^{\infty}=0,
$$

as reported in Section 2. 


\section{Conclusions}

One of our major concerns in working out these elementary models in complete detail has been to appraise the stationary-phase approximation (which here means completely discarding the nonperiodic closed orbits) and the approximation of ignoring all nonperiodic orbits except the shortest ones (which inevitably leads to a comparison with the problem on the half-line). Let us summarize the observations, in reverse order.

For the local vacuum energy density, the contribution of a short orbit is equal to the corresponding term in the half-line problem (restricted to the interval, of course). These are good approximations to the "boundary" part of the exact answer near the corresponding boundary, which is the only place where they are large. These boundary terms are not zero or generally small, so the stationary-phase approximation is not very good here.

For the local spectral density, or its unnamed indefinite integral, again the short orbit's contribution is equal to the half-line expression. Comparison with the Dirichlet kernel again suggests that these are good approximations near the boundary, and that these boundary terms are not very important elsewhere - although in this case they decay by virtue of oscillation (distributionally) more than by decrease of magnitude.

For the total vacuum energy, globally renormalized, we found that the boundary terms in both the exact answer and the half-line expression are equal to zero; that is, the stationary-phase approximation is exact! The short orbits, however, give a nonzero result; it is of roughly the same order of magnitude as the exact Casimir energy coming from the periodic orbits, so it must be rejected as wrong. The apparent contradiction with the local result is explained by the difference between local and global renormalization.

For the eigenvalue density, or its integral the counting function, the half-line theory and the exact theory agree (when both endpoints are counted), and they give the correction to the stationary-phase theory that is necessary to take account of the effect of the boundary conditions on the position of the lowest eigenvalue. The short-orbit expression differs, but it seems to be a reasonable approximation.

\section{Acknowledgements}

This work has been supported by the National Science Foundation under Grants DMS-0405806 and PHY-0554849.

\section{References}

[1] Balian R., Bloch C., Distribution of eigenfrequencies for the wave equation in a finite domain. III. Eigenfrequency density oscillations, Ann. Physics 69 (1972), 76-160.

[2] Balian R., Bloch C., Solution of the Schrödinger equation in terms of classical paths, Ann. Physics 85 (1974), 514-545.

[3] Balian R., Duplantier B., Electromagnetic waves near perfect conductors. I. Multiple scattering expansions. Distribution of modes, Ann. Physics 104 (1977), 300-335.

Balian R., Duplantier B., Electromagnetic waves near perfect conductors. II. Casimir effect, Ann. Physics 112 (1978), 165-208.

[4] Bär C., Moroianu S., Heat kernel asymptotics for roots of generalized Laplacians, Internat. J. Math. 14 (2003), 397-412.

[5] Bender C.M., Hays P., Zero-point energy of fields in a finite volume, Phys. Rev. D 14 (1976), $2622-2632$.

[6] Bernasconi F., Graf G.M., Hasler D., The heat kernel expansion for the electromagnetic field in a cavity, Ann. Henri Poincaré 4 (2003), 1001-1013, math-ph/0302035.

[7] Bordag M., Mohideen U., Mostepanenko V.M., New developments in the Casimir effect, Phys. Rep. 353 (2001), 1-205, quant-ph/0106045. 
[8] Boyer T.H., Van der Waals forces and zero-point energy for dielectric and permeable materials, Phys. Rev. A 9 (1974), 2078-2084.

[9] Brack M., Bhaduri R.K., Semiclassical physics, Addison-Wesley, Reading, 1997.

[10] Branson T.P., Gilkey P.B., The asymptotics of the Laplacian on a manifold with boundary, Comm. Partial Differential Equations 15 (1990), 245-272.

[11] Brown L.S., Maclay G.J., Vacuum stress between conducting plates: an image solution, Phys. Rev. 184 (1969), 1272-1279.

[12] Brownell F.H., Extended asymptotic eigenvalue distributions for bounded domains in $n$-space, J. Math. Mech. 6 (1957), 119-166.

[13] Casimir H.B.G., On the attraction between two perfectly conducting plates, Konink. Nederl. Akad. Weten., Proc. Sec. Sci. 51 (1948), 793-795.

[14] Cognola G., Vanzo L., Zerbini S., Regularization dependence of vacuum energy in arbitrarily shapted cavities, J. Math. Phys. 33 (1992), 222-228.

[15] Colin de Verdière Y., Spectre du laplacien et longuers des géodésiques périodiques. II, Compos. Math. 27 (1973), 159-184.

[16] Combescure M., Ralston J., Robert D., A proof of the Gutzwiller semiclassical trace formula using coherent states decomposition, Comm. Math. Phys. 202 (1999), 463-480, math-ph/9807005.

[17] DeWitt B.S., Quantum field theory in curved spacetime, Phys. Rep. 19 (1975), 295-357.

[18] Dowker J.S., 1. The counting function. 2. Hybrid boundary conditions, Nuclear Phys. B Proc. Suppl. 104 (2002), 153-156.

[19] Duistermaat J.J., Guillemin V.W., The spectrum of positive elliptic operators and periodic bicharacteristics, Invent. Math. 29 (1975), 39-79.

[20] Estrada R., The Cesáro behavior of distributions, Proc. Roy. Soc. London A 454 (1998), 2425-2443.

[21] Estrada R., Fulling S.A., Distributional asymptotic expansions of spectral functions and of the associated Green kernels, Electron. J. Differential Equations 1999 (1999), 07, 37 pages, funct-an/9710003.

[22] Estrada R., Fulling S.A., Functions and distributions in spaces with thick points, in Special Issue for the Tricentennial of Leonard Euler, Internat. J. Appl. Math. Stat., to appear, available at this URL http://www . math.tamu.edu/ fulling/fatpts4.pdf.

[23] Estrada R., Fulling S.A., Kaplan L., Kirsten K., Liu Z.H., Milton K.A., Classical paths and quantum vacuum stress in rectangular cavities, in preparation.

[24] Ford L.H., Svaiter N.F., Vacuum energy density near boundaries, Phys. Rev. D 58 (1998), 065007, 8 pages, quant-ph/9804056.

[25] Fulling S.A., The local geometric asymptotics of continuum eigenfunction expansions I, SIAM J. Math. Anal. 13 (1982), 891-912.

[26] Fulling S.A., Systematics of the relationship between vacuum energy calculations and heat kernel coefficients, J. Phys. A: Math. Gen. 36 (2003), 6857-6873, quant-ph/0302117.

[27] Fulling S.A., Global and local vacuum energy and closed orbit theory, in Quantum Field Theory under the Influence of External Conditions (6th Workshop ..., Norman, 2003). Editor K.A. Milton, Rinton Press, Princeton, 2004, 166-174, available at this URL http://www.math.tamu.edu/ fulling/funorman.pdf.

[28] Fulling S.A., Gorbar E.V., Romero C.T., Spectral Riesz-Cesàro means: How the square root function helps us to see around the world, in Mathematical Physics and Quantum Field Theory (E.H. Wichmann Symposium, Berkeley, 1999), Electron. J. Differ. Equ. Conf. 04 (2000), 87-101, available at this URL http://ejde.math.unt.edu/conf-proc/04/f3/abstr.html.

[29] Fulling S.A., Gustafson R.A., Some properties of Riesz means and spectral expansions, Electron. J. Differential Equations 1999 (1999), 06, 39 pages, physics/9710006.

[30] Fulling S.A., Wilson J.H., Vacuum energy and closed orbits in quantum graphs, in Program on Analysis on Graphs and Its Applications (Isaac Newton Institute, Cambridge, 2007), Editor P. Kuchment, to appear.

[31] Gilkey P.B., The spectral geometry of the higher order Laplacian, Duke Math. J. 47 (1980), 511-528.

[32] Gilkey P.B., Invariance theory, the heat equation and the Atiyah-Singer index theorem, CRC Press, Boca Raton, 1995.

[33] Gilkey P.B., Grubb G., Logarithmic terms in asymptotic expansions of heat operator traces, Comm. Partial Differential Equations 23 (1998), 777-792. 
[34] Gradshteyn I.S., Ryzhik I.M., Table of Integrals, series, and products, 5th ed., Academic Press, New York, 1980.

[35] Greiner P., An asymptotic expansion for the heat equation, Arch. Ration. Mech. Anal. 41 (1971), 163-218.

[36] Gutzwiller M.C., Periodic orbits and classical quantization conditions, J. Math. Phys. 12 (1971), 343-358 (and related papers).

[37] Hays P., Vacuum fluctuations of a confined massive scalar field in two dimensions, Ann. Physics 121 (1979), $32-46$.

[38] Helson H., Harmonic analysis, Addison-Wesley, Reading, 1983.

[39] Hertzberg M.P., Jaffe R.L., Kardar M., Scardicchio A., Casimir forces in a piston geometry at zero and finite temperatures, arXiv:0705.0139.

[40] Hörmander L., The spectral function of an elliptic operator, Acta Math. 121 (1968), 193-218.

[41] Iannuzzi D., Lisanti M., Munday J.N., Capasso F., The design of long-range quantum electrodynamical forces and torques between macroscopic bodies, Solid State Commun. 135 (2005), 618-626.

[42] Isham C.J., Twisted quantum fields in a curved space-time, Proc. Roy. Soc. London A 362 (1978), 383-404.

[43] Jaekel M.T., Reynaud S., Casimir force between partially transmitting mirrors, J. Physique I 1 (1991), 1395-1409.

[44] Kac M., Can one hear the shape of a drum?, Amer. Math. Monthly 73 (1966), Part II, 1-23.

[45] Kirsten K., Spectral functions in mathematics and physics, Chapman \& Hall/CRC, Boca Raton, 2002.

[46] Lebedev S.L., Casimir effect in the presence of an elastic boundary, Zh. Eksp. Teor. Fiz. 110 (1996), 769-792 (English transl.: JETP 83 (1996), 423-434).

[47] Littlejohn R.G., The Van Vleck formula, Maslov theory, and phase space geometry, J. Stat. Phys. 68 (1992), $7-50$.

[48] Lukosz W., Electromagnetic zero-point energy shift induced by conducting closed surfaces, Z. Physik 258 (1973), 99-107.

[49] Mazzitelli F.D., Sánchez M.J., Scoccola N.N., von Stecher J., Casimir interaction between two concentric cylinders: exact versus semiclassical results, Phys. Rev. A 67 (2003), 013807, 11 pages, quant-ph/0209097.

[50] Milton K.A., The Casimir effect: physical manifestations of zero-point energy, World Scientific, Singapore, 2001.

[51] Minakshisundaram S., Eigenfunctions on Riemannian manifolds, J. Indian Math. Soc. 17 (1953), $159-165$.

[52] Plunien G., Müller B., Greiner W., The Casimir effect, Phys. Rep. 143 (1986), 87-193.

[53] Romeo A., Saharian A.A., Casimir effect for scalar fields under Robin boundary conditions on plates, J. Phys. A: Math. Gen. 35 (2002), 1297-1320.

[54] Scardicchio A., Jaffe R.L., Casimir effects: an optical approach. I. Foundations and examples, Nuclear Phys. B 704 (2005), 552-582, quant-ph/0406041.

Scardicchio A., Jaffe R.L., Casimir effects: an optical approach. II. Local observables and thermal corrections, Nuclear Phys. B $\mathbf{7 4 3}$ (2006), 249-275, quant-ph/0507042.

[55] Schaden M., Sign and other aspects of semiclassical Casimir energies, Phys. Rev. A 73 (2006), 042102, 16 pages, hep-th/0509124.

[56] Schaden M., Semiclassical electromagnetic Casimir self-energies, hep-th/0604119.

[57] Schaden M., Spruch L., Infinity-free semiclassical evaluation of Casimir effects, Phys. Rev. A 58 (1998), 935-953.

[58] Stewart J., Calculus, 3rd ed., Brooks/Cole, Pacific Grove, 1995.

[59] Wilson J.H., Vacuum energy in quantum graphs, Undergraduate Research Fellow Thesis, Texas A\&M University, 2007, available at this URL http://handle.tamu.edu/1969.1/5682.

[60] Wolfram S., The Mathematica book, 4th ed., Cambridge University Press, Cambridge, 1999. 